\title{
Fluorescent Chemosensor for Quantitation of Multiple Atmospheric Gases
}

\section{Bhopate DP ${ }^{1,3 *}$, Kim K-H², Mahajan PG ${ }^{1}$, Gore AH ${ }^{1}$, Patil SR ${ }^{1}$, Majhi SM ${ }^{3}$, Naik GK ${ }^{3}$, Liang T-T ${ }^{3}$, Ahemad J-Md ${ }^{3}$, Yu Y-T $^{3}$ and Kadam AN ${ }^{4 *}$}

${ }^{1}$ Sharad Institute of Technology College of Engineering, (Yadrav) Ichalkaranji,, Shivaji University Kolhapur, Maharashtra, India ${ }^{2}$ Department of Civil and Environmental Engineering, Hanyang University, 222Wangsimni-Ro, Seoul Korea

${ }^{3}$ Division of Advanced Materials Engineering and Research Centre for Advanced Materials Development, Chonbuk National University, Jeonju 561-756, South Korea ${ }^{4}$ Department of Chemical and Biochemical Engineering, Gachon University Seongnam City, South Korea 461-701

\begin{abstract}
Recently, the sensing and monitoring of gases from ambient as well as industrial sources has gained a great importance in order to ensure occupational hygiene, public health, and societal welfare. The development of new technologies for visualizing and detecting gases at trace levels is imperative for various applications. There exist several established traditional methods to detect different gases. In this article, we review the latest trends in the area of fluorescence sensing of gas molecules, which is a high sensitivity technique with minimum or negligible interferences. The gas sensors fabricated with the use of fluorescent nanoparticles as detecting elements possess special feature, like high surface-to-volume ratios, ultra sensitivity, enhanced selectivity, cost effectiveness, and fast response. The inherent properties of the related systems, e.g. a large fluorescence lifetime, nanoscale particle size and a tunable zeta potential, make it possible to devise fluorescent sensors with an attractive pathway of fluorescence 'off-on'. Several fluorimetric methods are known to detect specific gases from the atmospheric gaseous samples with satisfactory detection results. Modern fluorescent gas sensors are did not cause interference from the co-pollutants thus making the fluorimetric sensing process to be quantitative as well as specific.
\end{abstract}

Keywords: Fluorescent gas sensor; Fluorescent organic nanoparticles, Fluorescence lifetime, Job's plot; Analytical method; Atmospheric environmental sample

\section{Introduction}

A chemical sensor essentially consists of transducer for converting the chemical information into diverse electronic or optical signals like frequency current, voltage, color, fluorescence and surface plasmon resonance [1-4]. Gas sensors are of paramount importance, as they allow much convenient and routine detections of several gases/vapors which can be hazardous to human health [5-7]. Over the past several decades various sensing technologies have been developed and employed for the detection of different gases. The underlying mechanisms are based on the measurement of properties, such as catalytic, electrochemical, optical, acoustic and conductivity. The performance characteristics of gas sensor have been evaluated in terms of important performance parameters, including sensitivity, selectivity, detection limit, response time and recovery time [8].

Electrochemical (EC) and Infrared spectroscopy (IR) methods have been the common choices for the sensing gases [9-12]. EC sensors are power-hungry and commonly work at high temperatures (300$800^{\circ} \mathrm{C}$ ). Thus, they are unsuitable for use in potentially flammable and explosive locations. IR sensors, on the other hand, are bulky and expensive. Also, they face serious problem of interference from other gases, as different gases can absorb similar wavelengths in the IR spectral region. Moreover, both the EC and IR chemosensors are cross-sensitive to water. Another class of gas sensor exploits optical properties. The optical gas sensors are used in fire and explosion prevention, healthcare, indoor air quality control, leak detection, food production and process optimization and control, among the other applications [13]. This particulate technology has progressed rapidly and now-a-days the optical gas sensors are capable to monitor multiple gases, sometimes remotely at a distance of a kilometer or more. The optical sensors can also create 2- and 3-D distribution maps, detect concentrations to as low as parts per trillion (ppt) region and even visualize gas plumes in real time.

Several traditional sensing methods have been established to detect gas, such as catalytic, thermal, semiconductors, and surface acoustic wave, infrared spectroscopic, electrochemical and gas chromatographymass spectrometric (GC-MS) techniques [14-16]. Most of those conventional methods have complex and costly instrumentation and invariably need a long analysis time. In some of them, it is difficult to tolerate the interference of other gases and water $[17,18]$. Note that GCMS can also be used to analyze gas, but the process is also costly and time-consuming. Field-effect transistors, based on the use of carbon nanotubes and inorganic semiconductors are promising for gasdetection applications but these devices are difficult to be fabricated with a precise process control needed [19].

The development of fluorescence-based gas sensors has been driven because of their certain potential advantages over other sensing techniques [20-25]. The fluorescence-based gas sensors, provide simplicity, fast response, safe operation, real time on-line detection and possible immunity to the co-pollutant interference [26]. The related area of the research has translated up to a certain commercially viable option with the availability of a range of fluorescence based sensors for a variety of gases, including oxygen carbon-dioxide, hydrogen, carbon monoxide, nitrogen oxide, etc. The applications of fluorimetric sensor extend to the real-time measurement of gases in atmosphere and measurement of dissolved gases. These modern fluorescent sensors boast of significantly improved performance in terms of sensitivity and working concentration ranges as compared to the most of the above mentioned competing techniques.

The strategies and ideas that chemists have developed for new

*Corresponding author: Dhanaji Pandit Bhopate, Division of Advanced Materials Engineering and Research Centre for Advanced Materials Development, Chonbuk National University, Jeonju 561-756, South Korea, Tel: 010-3235-6693; E-mail: dhananjaibhopate@gmail.com

Received: March 15, 2017; Accepted: March 29, 2017; Published: April 05, 2017

Citation: Bhopate DP, Kim K-H, Mahajan PG, Gore AH, Patil SR, et al. (2017) Fluorescent Chemosensor for Quantitation of Multiple Atmospheric Gases. J Nanomed Nanotechnol 8: 436. doi: 10.4172/2157-7439.1000436

Copyright: @ 2017 Bhopate DP, et al. This is an open-access article distributed under the terms of the Creative Commons Attribution License, which permits unrestricted use, distribution, and reproduction in any medium, provided the original author and source are credited. 
fluorescent gas sensing materials are reviewed here. The review focuses mainly on the work done during the last 5 years. This review will be limited to the development of gas sensors based on new artificial materials that are able to signal reversibly the presence of other interfering species. Nevertheless, due to the fact this field is very broad; it is very difficult to give a detailed overview of every publication that has appeared in literature. None the less, all the information about the subject is included, sometimes by references to other reviews which are an exhaustive overview of a specific material type. Additionally, new trends in the development of fluorescent gas sensors such as the fabrication of nano gas sensor, and the fabrication of high density sensor arrays will be also briefly discussed.

\section{Methodology}

\section{Fluorimetric gas sensing technique}

Analysis of gases is commonly accomplished by several techniques like catalytic, thermal, semiconductors, surface acoustic wave and infrared spectroscopy, electrochemical and Gas chromatography mass spectrometry (GC-MS) techniques. As such, the fluorimetric gas sensing technique is prone to the interferences from water vapors. It may also require the use of long path lengths sample tubes and other expensive equipment. Even though the electrode, (or modified versions), still exists, they face some issue of high cost, complexity, interferences and slow response times. Due to ease of production and use, the application of the optical sensors have been demonstrated for many analytes. Most of the times, the fluorimetric gas sensor are inexpensive and can be mass-produced as disposable chemical sensors. Coupling the optical sensors with fiber optics gives an opportunity for remote sensing applications [26]. The optical sensors employ two basic types of signal transduction mechanisms i.e. colorimetric or luminescent.

Many features of the fluorescence as a transduction mechanisms make it one of the most powerful chemical recognition event [27]. Over the years, a number of fluorescence microscopy and spectroscopy techniques have been developed based on the life-time, anisotropy or intensity of the emission from the fluorescent probes $[28,29]$. These are enormously sensitive techniques that even allow the detection of single molecules. Fluorescence based methods do not consume analytes and there is also no particular requirement of a reference material. As light can travel without physical waveguide, it facilitates relatively simple technical requirements. Additionally, the fluorescence based sensors can provide data through remote monitoring [30].

Fluorimetry, the measurement and use of fluorescence, is a technique for the qualitative and quantitative chemical analysis. It is ideally suited to the on field use and also free from stray-light interferences which is a common problem in the colorimetric technique [31]. Fluorimetry is a fundamentally sensitive, specific, handy, simple, and economical technique [32,33]. As a result, fluorometry has found an extensive range of applications in the study and detection of pollutants, sensing of gas and biomolecules as well as a host of other analyte making use of fluorescent materials as tracers.

\section{Important gas sensing phenomenon: Aggregation Induced Emission (AIE) properties}

Conventional luminophores in gas sensing tend to form aggregates in aqueous solutions due to the high hydrophobicity of their emitting centers (often aromatic rings). Understanding light emission in the aggregate state is thus of great importance for their practical gas sensing application. Tang et al. first described a novel phenomenon opposite to the aggregation caused quenching (ACQ) effect: propeller-shaped molecules such as hexaphenylsilole (HPS) and tetraphenylethylene (TPE) emit strongly in the solid state but are weakly emissive in solution. The phenomenon is called aggregation induced emission (AIE) [34,35]. This rare observation prompted the authors to extend the study on luminescence behaviors. Also, the dilute ethanolic solution of 1-methyl-1,2,3,4,5-pentaphenylsilole (1) was excited at $381 \mathrm{~nm}$, nearly no fluorescence signals were measured. In contrast, when large amounts of water were added to its ethanol solutions (the final concentrations being kept unchanged at $10 \mathrm{mM}$ ), intense photoluminescence spectra were recorded under identical measurement conditions. As water could not solubilize the compound 1, the silole (Figure 1) molecules must have aggregated in the solvent mixtures with high water contents. The 'solutions' were, however, macroscopically homogenous with no precipitate, suggesting that the silole aggregates were of nanodimension.

The above explained phenomena of the AIE active materials could be having its potential utility in the sensing fields. The AIE-active molecules do not show emission in solutions but when aggregated or in the solid state, they show intense emission because of the prevention of intramolecular rotations that avoid nonradiative deactivation for fluorescence quenching. Based on the aggregation state dependent luminescence, AIE-active molecules can be used as an alternative sensing element for bimolecular species. As example of the fluorometric sensing the detection and identification of $\mathrm{CO}_{2}$ was suggested by using AIE-active HPS with carbamate ionic liquid (CIL) by bubbling $\mathrm{CO}_{2}$ gas through dipropylamine (DPA) liquid (Figure 2a) [34-38]. After being bubbled with $\mathrm{CO}_{2}$ gas, the HPS mixture emitted a very bright green light. Photoluminescence (PL) spectrum of HPS was intensified with
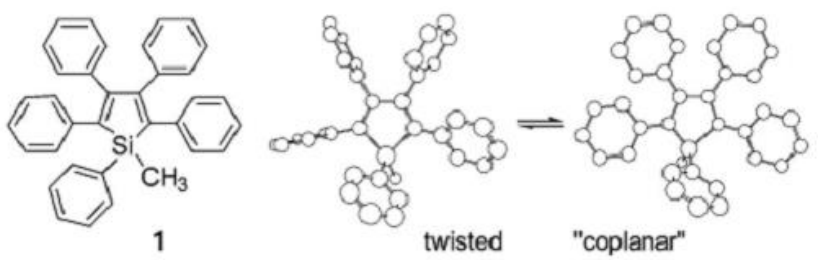

Figure 1: Molecular structure and conformational roamers of 1.
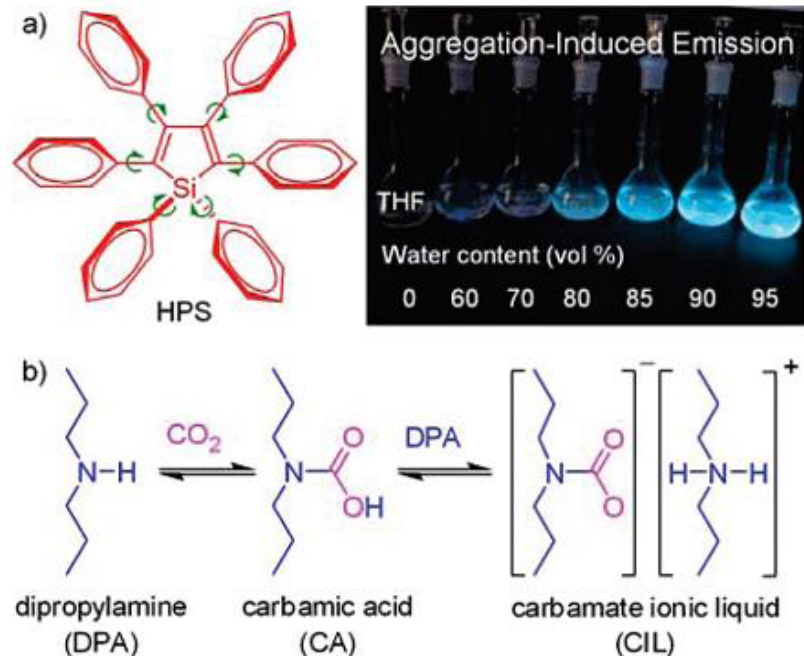

Figure 2a: Fluorometric sensing for detection and identification of $\mathrm{CO}_{2}$ by using AIE-active 1, 1, 2, 3, 4, 5-Hexaphenylsilole (HPS) 
Citation: Bhopate DP, Kim K-H, Mahajan PG, Gore AH, Patil SR, et al. (2017) Fluorescent Chemosensor for Quantitation of Multiple Atmospheric Gases. J Nanomed Nanotechnol 8: 436. doi: 10.4172/2157-7439.1000436

increasing the volume of $\mathrm{CO}_{2}$ gas. Therefore, it is concluded that HPS is acts as a fluorescent sensor for $\mathrm{CO}_{2}$ gas detection.

In another fluorimetric sensing example a composite between a flexible porous coordination polymer and fluorescent reporter distyrylbenzene (DSB) selectively adsorbed $\mathrm{CO}_{2}$ over other atmospheric gases. This adsorption induced a host transformation, which was accompanied by conformational variations of the included DSB. This read-out process resulted in a critical change in DSB fluorescence at a specific threshold pressure. The composite shows different fluorescence responses to $\mathrm{CO}_{2}$ and acetylene compounds that have similar physicochemical properties. Their system showed, for the first time, that fluorescent molecules can detect gases without any chemical interaction or energy transfer (Figure 2b) [39].

\section{Plausible materials}

Classical design of fluorescent gas indicators: The classical design of a fluorescent indicator includes two moieties, a receptor responsible for the molecular recognition of the analyte and a fluorophore responsible for signaling the recognition event. There are three main strategies to approach the design of fluorescent molecular indicators for chemical sensing in solution. The first results in intrinsic fluorescent probes, which are fluorescent molecules where the mechanism for signal transduction involves interaction of the analyte with a ligand that is part of the $\pi$-system of the fluorophore. The second are extrinsic fluorescent probes, in which the receptor moiety and the fluorophore are covalently linked but are electronically independent. These approaches involve different receptor molecules that might be synthesized and afterwards attached to a fluorophore to make the sensitive probe. Due to the covalent linking through a spacer the moieties are in close proximity, and thus interaction of the analyte with the receptor induces a change in the fluorophore surroundings and alters its fluorescence. The third strategy is called chemical ensemble. This approach is based on a competitive assay in which a receptorfluorophore ensemble Design of Fluorescent Materials for Chemical Sensing is selectively dissociated by the addition of an appropriate competitive analyte that is able to interact efficiently with the receptor, and resulting in a detectable response of the fluorophore.

Fluorescent materials for gas sensing: In order to make the fluorescent probes specific for the molecular target and applicable in the complex environment, reasonable imagination and scientific design should follow certain principles. Significantly, the first step in order to choose an appropriate reporter or fluorophore is crucial for the whole system. Fluorophores are divided into different classes according to their sensing properties and emission wavelength. A variety of fluorophores have been proposed as framework for the chemosensor design strategy, which may include anthracene 1, coumarin 2, naphthalimine 3, BODIPY 4 , fluorescein 5 , rhodamine 6 and cyanin 7 (Table 1 and Figure 3). All these fluorophores have been widely employed and applied as reporters in each systematic structure due to the various choice of emission wavelength [40].

The fluorescent probes may be designed on the basis of several underlying working principles, such as PET (photo induced electron transfer), ICT (internal charge transfer), FRET (Förster resonance energy transfer), and TICT (twisted internal charge transfer) (Figure 4) [41]. A typical fluorescent probe is fundamentally composed of a receptor (responsible for the molecular recognition of the gas), a signal reporter (fluorophore) responsible of signaling the recognition event and sometimes a spacer (for signal transformation with the target as signal input). Design of fluorescent indicators can be divided into three

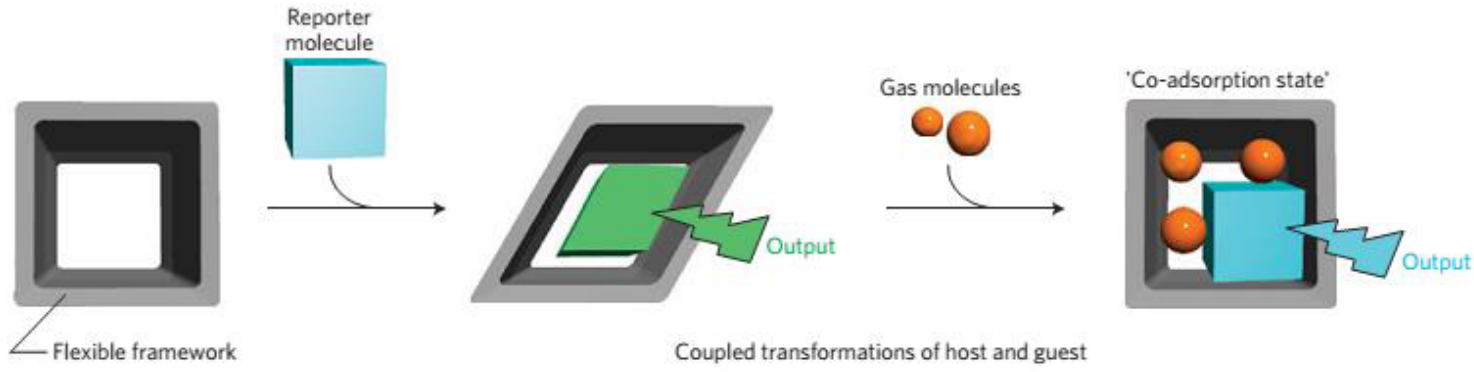

Figure 2b: Schematic illustration for detection of gas molecules by coupled structural transformations of a flexible PCP framework and a reporter molecule.

\begin{tabular}{|c|c|c|c|c|c|}
\hline Sr. No. & Authors & Fluorescence response & Gases detected & Substrate material & Detection limit \\
\hline 1 & Yun Ma [29] & $\begin{array}{l}\text { Fluorescence } \\
\text { enhancement }\end{array}$ & Carbon dioxide & Tetraphenylethene & $2.4 \times 10^{-6} \mathrm{M}$ \\
\hline 2 & Yang Liu [27] & $\begin{array}{l}\text { Fluorescence } \\
\text { enhancement }\end{array}$ & Carbon dioxide & 1,1,2,3,4,5-Hexaphenylsilole & In ppm \\
\hline 3 & Xin-Qi Zhan [51] & Fluorescence quenching & Nitrogen oxides & $\begin{array}{l}\text { 4,4,4,4-Tetra substituted amino aluminum } \\
\text { phthalocyanine }\end{array}$ & $0.08 \mathrm{ng} \mathrm{ml}^{-1}$ \\
\hline 4 & Wenying XU/1994 & Fluorescence quenching & Oxygen & $\begin{array}{l}{\left[\mathrm{Ru}(\mathrm{Phzphen})_{3} 1 \mathrm{Cl}_{2}\left(\mathrm{Ph}_{2} \text { phen=4,7-5diphenyl- }\right.\right.} \\
1,10 \text {-phenanthroline })\end{array}$ & In ppm \\
\hline 5 & Velasco-Garcıa & Fluorescence quenching & Oxygen & $\begin{array}{l}\text { Ruthenium complex with different } \\
\text { concentrations of gelatin in organogel }\end{array}$ & $0.2 \mathrm{ppm}$ \\
\hline 6 & Gillanders [18] & $\begin{array}{l}\text { Fluorescence } \\
\text { enhancement }\end{array}$ & $\begin{array}{l}\text { The response of the sensor films to } \\
\text { vapors of TNT, DNT, RDX }\end{array}$ & BBEHP-PPV phenylene-vinylene & In ppm \\
\hline 7 & Amornthammarong [2] & & Ammonia & o-Phthaldialdehyde (OPA) and sulfite & $1.1 \mathrm{nM}$ \\
\hline 8 & Jing Wang [42] & $\begin{array}{l}\text { Carbon Monoxide Imaging } \\
\text { in Living Cells }\end{array}$ & $\begin{array}{l}\text { A Selective Fluorescent Probe for } \\
\text { Carbon Monoxide Imaging in Living } \\
\text { Cells }\end{array}$ & $\begin{array}{l}\text { Unique CO- binding selectivity of CooA, } \\
\text { a dimeric CO-sensing heme protein from } \\
\text { Rhodospirillum rubrum }\end{array}$ & $\mu \mathrm{M}$ \\
\hline
\end{tabular}

Table 1: Review of fluorimetric gas sensor. 
main strategies for chemical sensing in solution. They are "intrinsic fluorescent probes" with a ligand as part of the $\pi$-system of the fluorophore; "extrinsic fluorescent probes" with electrical independence of the receptor moiety and the reporter which are covalently linked by a spacer; "chemosensor ensemble" based on the dissociation of receptorreporter ensemble selectively by the competitive analyte (Table 2).

Preparation of nanomaterials: Why nanomaterials?: The major classes of organic materials such as some polymer, lipid, dyes, polynuclear aromatic hydrocarbon and heterocyclic compound can selectively target gases such as $\mathrm{CO}_{2}, \mathrm{NOx}$ and $\mathrm{NH}_{3}$ [42-45]. Fluorescent organic nanoparticles are one of the most promising materials proposed for the gas sensing purpose due to the more flexibility in material synthesis and excellent optical properties. The organic nanoparticles are known to exhibit interesting phenomena, including photocatalytic activity, and enhanced photo induced charge separation. Their ability to sense and detect the chemicals and gas molecules result into the realization of new sensor strategies. Fluorescent organic nanoparticles have been employed to detect metal ions, anions, gases and biomolecules [46-49]. The aqueous suspension of organic nanoparticles prepared from simple reprecipitation method acts as excellent chemosensor

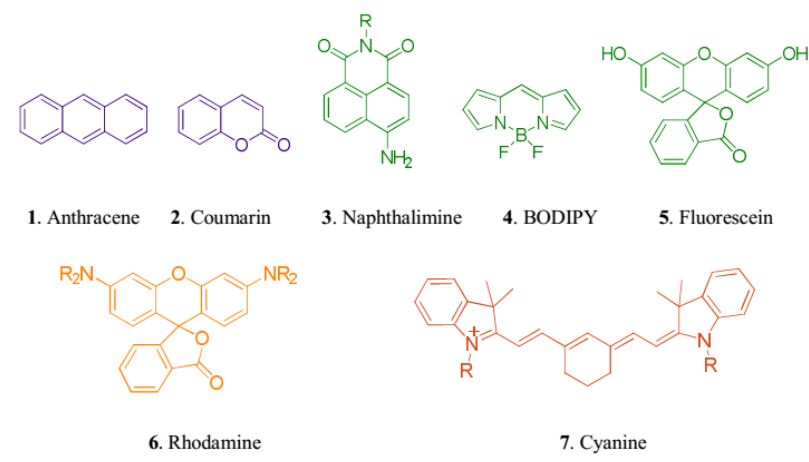

Figure 3: Structures of various luminophores.

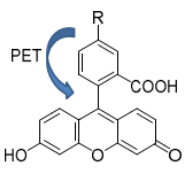

7. $\mathrm{R}=\mathrm{NO}_{2}$

8. $\mathrm{R}=\mathrm{NH}_{2}$

Figure 4: Various mechanism for fluorescent probe design.
[50,51]. The fluorescent organic nanoparticles may target the analyte gas molecules via binding through the functional groups presents on the nanoparticles surface or the surficial electrostatic attraction forces. These interactions generally result in the quenching of fluorescence signal. In the past few years have reported several material systems that have aggregation-induced enhanced emission (AIEE) properties (Park et al. [34]). The common structural characteristics of these AIEE active isolated molecules are twisted in conformation. The origin of the AIEE phenomenon is assumed to be related to the effects of intramolecular planarization or specific aggregation ( $\mathrm{H}$ - or J-aggregation (Figure $5 \mathrm{a}$ ) in the aqueous suspension of organic nanoparticles. These intramolecular vibrational and rotational motions, which behave strongly in these twisted conjugated molecules, lead to the fast nonradiative relaxation and reduced fluorescence quantum yield in solutions. Therefore the rigorous restriction of these free motions in the nano aggregated state is an approach to increase the fluorescence emission of AIEE active organic nanomaterials. Therefore these AIEE active organic nanomaterials acts as an excellent sensors.

Organic nanoparticles (ONPs) can be synthesized by different approach such as emulsification evaporation, emulsification diffusion, and solvent displacement methods [52]. The reprecipitation method (Figure 5b) which is a simple, quick, less expensive and green approach mostly used for the preparation of organic nanoparticles [36]. There are numerous examples on the use of the functionalized small organic nanoparticles for the sensing of various gas molecules of interest [42].

\section{Description of Sensor}

\section{Particle size distribution and morphological studies of nanosensor}

The particle size distribution histogram of ONPs aqueous suspension is generally studied with Dynamic Light Scattering (DLS) technique. The DLS investigations give an insight about the size distribution and colloidal stability of nanoparticles [30]. The measured data may also include contributions from various variables, such as surface coating and size differences. Figure 6 shows the particles size of indigo nanoparticle, giving a mean size distribution of $107 \mathrm{~nm}$ [37]. Some other example of DLS characterization includes the determination of perylene nanoparticles size, protein size, latex size, and colloid size [32-36]. In general, the DLS technique is best suited for submicron particles. However, with modern spectroscopic instrumentation, it has also been possible to use the DLS technique for particle with sizes lesser than a nanometer.

Scanning electron microscopy (SEM) is another technique used for the characterization of the ONPs. It gives information on the air dried layers of the ONPs (Figure 7). High-resolution scanning electron

\begin{tabular}{|c|l|l|}
\hline Sr. No. & \multicolumn{1}{|c|}{ Target gas } & Plausible fluorescent sensor material \\
\hline 1 & Carbon dioxide $\mathrm{CO}_{2}$ & $\begin{array}{l}\text { It is known that bubbling } \mathrm{CO}_{2} \text { through an amine yields a carbamate ionic liquid, which is accompanied by increases in polarity } \\
\text { and viscosity which results in turn on the light emission of amine group containing fluorescent sensor. }\end{array}$ \\
\hline 2 & Nitrogen dioxide $\left(\mathrm{NO}_{2}\right)$ & $\begin{array}{l}\text { Dye } \mathrm{N}, \mathrm{N}, \mathrm{N}^{\prime}, \mathrm{N}^{\prime} \text {-tetramethyl-p-phenylenediamine use as a sensor for the detection of } \mathrm{NO}_{2} \text {. Oxidation of dye with } \mathrm{NO}_{2} \text { leads to the } \\
\text { changes in fluorescence intensity results in development of fluorescent sensor. }\end{array}$ \\
\hline 3 & Carbon monoxide $(\mathrm{CO})$ & $\begin{array}{l}\text { The reaction of a binuclear rhodium complex is used for the CO reaction: the CO reaction takes place through a bound acetate } \\
\text { groups led to changes in fluorescent properties of rhodium complex. }\end{array}$ \\
\hline 4 & Ammonia $\left(\mathrm{NH}_{3}\right)$ & $\begin{array}{l}\text { Fraunhofer IPM uses bromophenol blue for fluorimetric detection of ammonia }\left(\mathrm{NH}_{3}\right) . \text { The acid-base reaction between these splits off } \\
\text { the proton of the hydroxy group. The protonated form then has a different fluorescence intensity than the deprotonated form. }\end{array}$ \\
\hline 5 & Oxygen & $\begin{array}{l}\text { Virtually all luminescence-based oxygen sensors use the quenching of the dye's luminescence by oxygen as the transduction } \\
\text { mechanism (fluorescence quenching). }\end{array}$ \\
\hline 6 & Explosives vapor detection & $\begin{array}{l}\text { A number of light-emitting polymer (poly(para-phenylenevinylene) (PPV) and polyfluorene backbones) sensor materials15 to a } \\
\text { selection of explosives and related materials including TNT, 2,4-DNT etc. }\end{array}$ \\
\hline
\end{tabular}

Table 2: Plausible fluorescent material for multiple gas detection. 

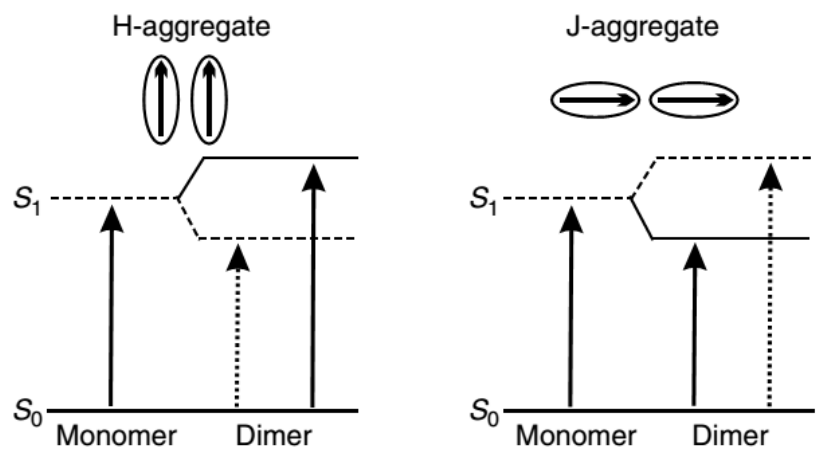

Figure 5a: Simplified schematic of exciton theory to explain the different absorption and fluorescence behaviors of $\mathrm{H}$ - and J-aggregates.

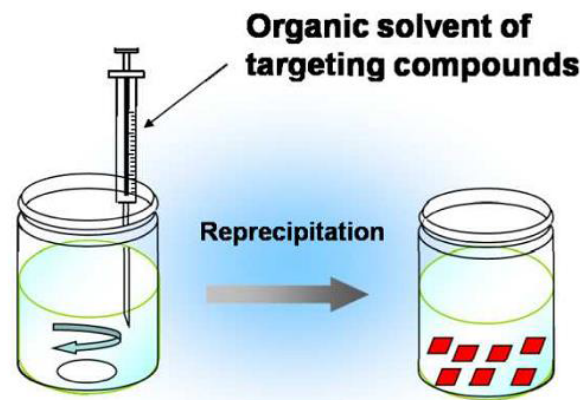

\section{Poor solvent: Water \\ Organic nanocrystals dispersed in water}

Figure 5b: The scheme of the reprecipitation method.

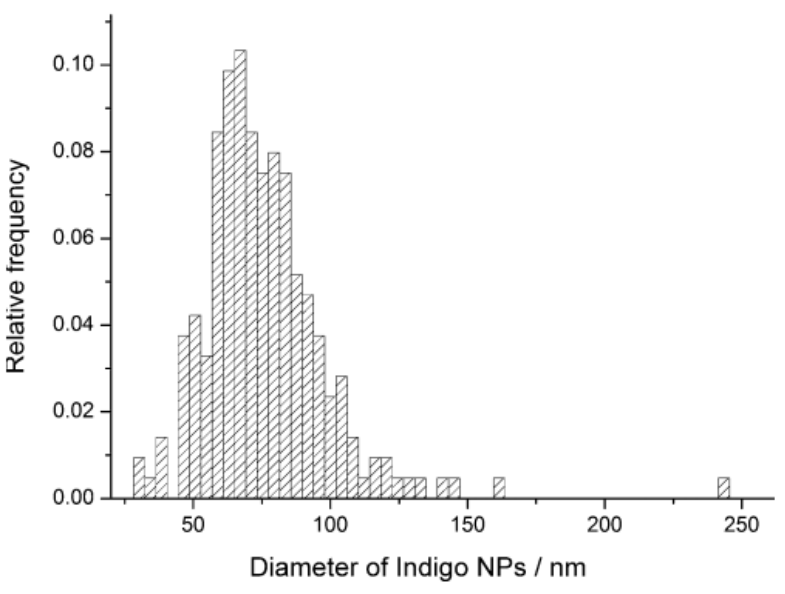

Figure 6: Distribution of the indigo nanoparticles diameter.

microscopes (SEM) as well as the transmission electron microscope are very important characterization tools for studying the morphology of the nanoparticles [42].

Optical absorption properties by UV-visible absorption spectroscopy

Absorption spectroscopy, in general, refers to characterization

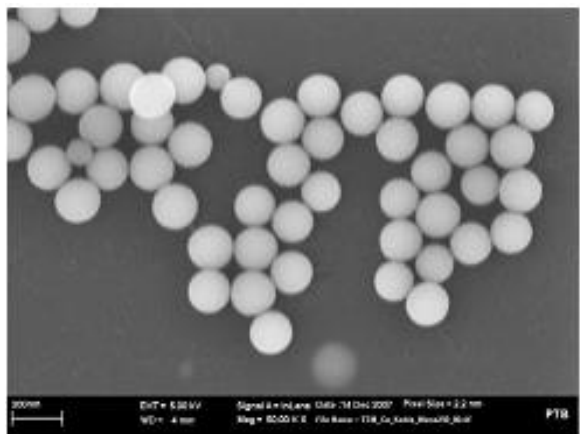

Figure 7: Typical SEM image of silica nanoparticles.

technique that measures the absorption of radiation by a material, as a function of the wavelength.

Absorption spectrum: It shows the dependence of the degree of light absorption by the compound. The quantization condition for the absorption or emission of light by an atom or by molecule is given by the Einstein relation as given by equation [44],

$$
\begin{aligned}
& E=h v=\frac{h c}{\lambda} \\
& h v=\frac{h c}{\lambda}=E_{2}-E_{1}
\end{aligned}
$$

Where $\mathrm{E}_{2}$ and $\mathrm{E}_{1}$ are the electronic energy levels.

The absorption of energy by a molecule is governed by the BeerLambert's law. According to this relationship,

$$
\log _{10}\left(\frac{I_{0}}{I}\right)=\text { E.c.l }
$$

Where $I_{0}$ - intensity of incident light,

I: Intensity of transmitted light,

$\varepsilon$ : Molecular extinction coefficient,

$c$ : Concentration of the path length,

$l$ : Path length of the absorbing system through which light passes, and $\log _{10}\left(\frac{I_{0}}{I}\right)$ - optical density or absorbance of the material.

In general, the absorption spectrum is plotted in terms of the molecular extinction coefficient $(\varepsilon)$ against frequency or wavelength The probability of the absorption depends upon the degree of overlap of the wave function of the lowest vibrational level of the ground state and the wave function of the vibrational level of the first excited singlet state $S_{10} \rightarrow S_{1 n}$. The positions of the absorption peaks and its nature are of significance in the spectroscopic studies. In solution, the broad absorption band is an indication of dimeric nature of molecules in the ground state while the structured spectrum indicates the existence of monomolecular species, but in solids the absorption spectra are not as structured as in solution. The nature of absorption band also gives an idea about the lattice structures of molecular system under study.

\section{Emission spectrum}

Emission spectroscopy, in general, refers to a characterization technique that measures the emission of radiation by a material that has been excited. Fluorescence spectroscopy is one type of emission spectroscopy which records the intensity of light radiated from 
the material as a function of wavelength [28]. It is a nondestructive characterization technique. After an electron is excited from the ground state, it needs to relax back to the ground state. This relaxation or loss of energy to return to the ground state can be achieved by a combination of non-radiative decay (loss of energy through heat) and radiative decay (loss of energy through light). Emission spectrum defines the relative intensity of radiation emitted at various wavelengths [44]. The emitted light comprises fluorescence, delayed fluorescence and phosphorescence, thereby yielding three types of emission spectra. The Fluorescence emission spectrum shows an almost mirror like symmetry with its absorption. The fluorescence emission spectrum is obtained by irradiating the sample by a wavelength of maximum absorption. The ground state and excited state are associated with the absorption and emission spectra. It is observed that the absorption spectra gives data about the vibrational levels of the excited state and the emission spectra yield data about the vibrational levels of the ground state.

The same fluorescence emission spectrum is generally observed irrespective of the excitation wavelength. Upon excitation into higher electronic and vibrational levels, the excess energy is quickly dissipated, leaving the molecule in the lowest vibrational level of $\mathrm{S}_{1}$. This relaxation occur in about $10^{-12}$ second and is presumable a result of strong overlap among numerous states of nearly equal energy. Because of this rapid relaxation, emission spectra are usually independent of the excitation wavelength.

\section{Excitation spectrum}

It defines as the relative efficiency of different wavelengths of exciting radiation to induce fluorescence. The excitation spectrum is obtained by measuring the fluorescence intensity at a fixed emission wavelength while the excitation wavelength is scanned. For large, complex molecules, the excitation spectrum is quite stable, independent of the emission wavelength. The excitation spectrum will be identical to the absorption spectrum where $\varepsilon . c . l<<1$. The measurement of quantum intensity is limited by the sensitivity of the spectrofluorimeter and that depends upon the intensity of the excitation source. Crank et al. [11] estimated that concentrations as low as $10^{-12} \mathrm{~mol} . \mathrm{dm}^{-3} \mathrm{can}$ be detected by excitation spectroscopy compared with a minimum concentration of $10^{-8} \mathrm{~mol} . \mathrm{dm}^{-3}$ by absorption spectroscopy [28]. Excitation spectroscopy is also used to determine the quantum efficiency of energy transfer between donor and acceptor molecules.

\section{Fluorescence lifetime}

Time-resolved fluorescence provides more information about the molecular environment of the fluorophore than steady state fluorescence measurements. Since the fluorescence lifetime of a molecule is very sensitive to its molecular environment, measurement of the fluorescence lifetime(s) reveals much about the state of the fluorophore. Many macromolecular events, such as rotational diffusion, resonance-energy transfer, and dynamic quenching, occur on the same time scale as the fluorescence decay. Thus, time-resolved fluorescence spectroscopy can be used to investigate these processes and gain insight into the chemical surroundings of the fluorophore [28].

\section{Fluorescent Chemosensor for Selective Detection and Quantitation of Multiple Gases}

\section{Gas recognition test using fluorescent sensor}

Different gas contents were bubbled through sensor solutions at a fixed rate for a fixed time or vice-versa and observation of recognizable changes in the emission spectrum of fluorescent sensor.
Ex. Bubbling large volumes of $\mathrm{CO}_{2}$ gas through 1,2,3,4,5-Hexaphenylsilole (HPS) solutions in diethylamine (DEA), butylamine (BA), piperidine (Pip), and pyridine (Py) caused no recognizable changes in the emission of HPS. Photoluminescence (PL) spectrum of HPS was intensified with increasing the volume of $\mathrm{CO}_{2}$ gas. After being bubbled with $25 \mathrm{~mL}$ of $\mathrm{CO}_{2}$ gas, the HPS mixture emitted a very bright green light [19-25].

\section{Selectivity of recognition of gas (specific) by sensor}

Specific gas (sensed by sensor) with presence of different gas contents were bubbled through sensor solutions at a fixed rate for a fixed time and observation of recognizable changes in the emission spectrum of fluorescent sensor [20].

Ex. The fluorescence spectra of sensor with and without specific gas and that of other coexisting gas each of same concentration were recorded by monitoring particular excitation wavelength and the extent of fluorescence changes i.e. $\Delta \mathrm{F}=\mathrm{F}_{0}-\mathrm{F}$ and $\Delta \mathrm{F} / \mathrm{F}$ were calculated and graphically presented by bar diagram. ' $\mathrm{F}$ ' is the intensity of fluorescence of sensor in presence of specific gas and other interfering gases and $\mathrm{F}_{0}$ is the intensity without coexisting gases $[21,22]$.

\section{Fluorimetric titration of sensor with gas (specific) experiment}

The fluorescence sensing experiments involved measurement of fluorescence spectra of sensor with and without analyte gas sample. The solution of sensor and specific gas for bubbling at a fixed rate for a fixed time required in the experiment sets. An appropriate quantity of sensor solution was kept constant while specific gas for bubbling at a fixed rate for a fixed time varied from $\mathrm{mL}$ to $\mathrm{mL}[20,21]$.

Stern-Volmer plot: The fluorescence quenching is due to the binding interaction of analyte gas with the sensor. The quenching results fit into the conventional linear Stern-Volmer equation given below [25].

$$
\frac{F_{0}}{F}=1+k_{q} \tau_{0}[Q]=1+K_{S V}[Q]
$$

Where Fo and $\mathrm{F}$ are the fluorescence intensity of the sensor in the absence and in the presence of the analyte gas respectively. $\mathrm{kq}, \mathrm{K}_{\mathrm{sV}}$, $\tau_{\mathrm{o}}$ and $[\mathrm{Q}]$ are the quenching rate constant of the analyte gas, SternVolmer quenching constant, average fluorescence life time of sensor without analyte gas and concentration of analyte gas respectively.

For fluorescence enhancement a calibration curve was constructed by plotting $\Delta \mathrm{F}$ (increase in fluorescence intensity) versus the concentration of analyte gas.

Statistical analysis: (Limit of detection or quantitation and comparison with other method)

Limit of detection (LOD)

$\mathrm{LOD}=(3.3 \sigma / \mathrm{k})$

Where $\sigma$ is the standard deviation of the $y$-intercepts of regression lines and $\mathrm{k}$ is the slope of the calibration graph. After calculating LOD we compared our sensing method with existing method by preparing comparisons table (Table 3).

Stoichiometry and binding parameters of (Sensor+Gas (specific)): The ligand-metal ion stoichiometry was estimated by applying Job's method by keeping the sum of the concentration of the analyte and sensor constant and varying the molar fraction of analyte from 0.1 to 0.9 . 
Citation: Bhopate DP, Kim K-H, Mahajan PG, Gore AH, Patil SR, et al. (2017) Fluorescent Chemosensor for Quantitation of Multiple Atmospheric Gases. J Nanomed Nanotechnol 8: 436. doi: 10.4172/2157-7439.1000436

Page 7 of 9

\begin{tabular}{|c|c|c|c|c|c|}
\hline Sr. No. & Sensor Type & Target & Detection limit & Advantages & Disadvantages \\
\hline 1. & Catalytic & $\begin{array}{l}\text { Hydrogen, carbon monoxide, carbon } \\
\text { dioxide, nitrogen oxide, sulfur oxide, } \\
\text { ammonia }\end{array}$ & $\begin{array}{l}\text { lower explosive limit }(0- \\
100 \% \text { LEL) }\end{array}$ & $\begin{array}{l}\text { Simple, measures flammability of } \\
\text { gases and low cost technology }\end{array}$ & $\begin{array}{l}\text { Requires air or oxygen to work. } \\
\text { Can be poisoned by lead, } \\
\text { chlorine and silicones }\end{array}$ \\
\hline 2. & Thermal & $\begin{array}{l}\text { Hydrogen, carbon monoxide, nitrogen, } \\
\text { sulfur oxide, inorganic gases and } \\
\text { many other compounds }\end{array}$ & ppm & $\begin{array}{l}\text { Robust but simple construction. Easy } \\
\text { to operate in absence of oxygen. } \\
\text { Measuring range is very wide. }\end{array}$ & Reaction due to heating wire. \\
\hline 3. & Electrochemical & $\begin{array}{l}\text { Hydrogen, carbon monoxide, carbon } \\
\text { dioxide, nitrogen dioxide, ammonia }\end{array}$ & ppm & $\begin{array}{l}\text { Measures toxic gases in relatively low } \\
\text { concentrations. Wide range of gases } \\
\text { can be detected }\end{array}$ & $\begin{array}{l}\text { Failures modes are unrevealed } \\
\text { unless advanced monitoring } \\
\text { technique used. }\end{array}$ \\
\hline 4. & Infrared & $\begin{array}{l}\text { Carbon monoxide, carbon dioxide, } \\
\text { hydrocarbon gas }\end{array}$ & $\mathrm{ppm}$ or $\mathrm{mg} / \mathrm{m}^{3}$ & $\begin{array}{l}\text { Uses only physical technique. No } \\
\text { unseen failure modes. Can be used in } \\
\text { inert atmospheres. }\end{array}$ & $\begin{array}{l}\text { Not all gases have IR absorption. } \\
\text { Sequential monitoring is slower } \\
\text { on multi point analyzers and also } \\
\text { more user expertise required. }\end{array}$ \\
\hline 5. & Semiconductors & $\begin{array}{l}\text { Carbon monoxide, nitrogen oxide, } \\
\text { ammoniac, sulfurous gases, and } \\
\text { hydrocarbons as well as volatile } \\
\text { organic compounds }\end{array}$ & $\begin{array}{l}\text { The detection limit } \\
\text { depends on the } \\
\text { respective gas sensitive } \\
\text { material. }\end{array}$ & $\begin{array}{l}\text { Mechanically robust, works well at } \\
\text { constant high humidity condition. }\end{array}$ & $\begin{array}{l}\text { Susceptible to contaminants and } \\
\text { changes due to environmental } \\
\text { conditions. Non- linear response } \\
\text { effects complexity. }\end{array}$ \\
\hline 6. & $\begin{array}{l}\text { Surface Acoustic } \\
\text { Wave }\end{array}$ & $\begin{array}{l}\text { Hydrogen, carbon monoxide, carbon } \\
\text { dioxide, nitrogen dioxide, ammonia, } \\
\text { volatile organic compounds, Methanol, } \\
\text { ethanol, isopropyl, alcohol, acetone, } \\
\text { amine }\end{array}$ & $\mathrm{Hz} / \mathrm{ppm}$ & $\begin{array}{l}\text { Detect nerve and blister agents } \\
\text { Battery-less and could be used for } \\
\text { wireless applications. Could be placed } \\
\text { in harsh and rotating parts. }\end{array}$ & $\begin{array}{l}\text { Due to its small size there is } \\
\text { difficulty in handling during } \\
\text { fabrication process. }\end{array}$ \\
\hline \multirow[t]{3}{*}{7.} & Optical & & & & \\
\hline & i) Colorimetric & $\begin{array}{l}\text { Carbon dioxide, oxygen, ammonia and } \\
\text { nitrogen dioxide }\end{array}$ & $\mathrm{ppm} / \mathrm{ppb}$ & $\begin{array}{l}\text { Easy to operate in absence of oxygen. } \\
\text { Not affected by electromagnetic } \\
\text { interference. Monitoring area is very wide. }\end{array}$ & $\begin{array}{l}\text { Affected by ambient light } \\
\text { interference. }\end{array}$ \\
\hline & ii) Fluorimetric & $\begin{array}{l}\text { Carbon dioxide, carbon monoxide, } \\
\text { oxygen, ammonia, sulfurous gases } \\
\text { explosive vapors and nitrogen dioxide }\end{array}$ & $\begin{array}{l}\mathrm{ppm}, \mathrm{ppb}, \mathrm{nM}, \mu \mathrm{g} / \mathrm{ml} \text {, } \\
\mathrm{ng} / \mathrm{ml}\end{array}$ & $\begin{array}{l}\text { The sensing methods based on } \\
\text { fluorescence are more sensitive and } \\
\text { selective than the calorimetric method } \\
\text { with lowest detection limit. }\end{array}$ & $\begin{array}{l}\text { Does not affected by ambient } \\
\text { light interference. }\end{array}$ \\
\hline
\end{tabular}

Table 3: Comparison of gas detection sensor technologies.

The binding constant $\left(\mathrm{K}_{\mathrm{B}}\right)$ was evaluated using modified BenesiHildebrand (B-H) equation given below [19-24].

$$
\frac{1}{F-F_{0}}=\frac{1}{\left(F_{\max }-F_{0}\right)}+\frac{1}{K_{B}\left(F_{\max }-F_{0}\right)} \frac{1}{[G a s]^{n}}
$$

Where $\mathrm{F}_{\max }$ is the maximum fluorescence intensity of sensor in presence of analyte gas, and $\mathrm{F}_{0}$ and $\mathrm{F}$ in absence and in the presence of analyte gas respectively. Gas represents the concentration of gas and ' $n$ ' is the number of gas molecules bound per sensor molecules.

Mechanism of binding interaction of Sensor+Gas (specific): Ground state complex formation does not change lifetime of fluorescent sensor with addition gas analyte. The decreases/increases in lifetime of sensor seen with increases in concentration of gas analyte supports the excited state complex formation.

Adsorption of gas molecules and its complexation with sensors is also confirmed by DLS and zeta potential measurements. The increases in particle size and zeta potential of sensor estimated after the sequential addition of gas molecule indicating adsorption of gas type of interaction.

The binding parameters like binding constant and number of binding sites becomes important while explaining the binding mechanism between two different molecules and they can be determined by following equation [21-25],

$$
\log \frac{F_{0}-F}{F}=\log K+n \log [Q]
$$

Where $\mathrm{F}$ and $\mathrm{F}_{0}$ are the fluorescence intensity in the presence and absence of quencher respectively, $K$ is the binding constant, $n$ is the number of binding sites and $[Q]$ is the concentration of quencher.

The binding constant $(K)$ and the number of binding sites $(n)$ can be calculated by plotting the graph of $\log \frac{F_{0}-F}{F}$ versus $\log [Q]$. The nature of this plot is a straight line with, intercept on Y-axis. The slope determines the number of binding sites while intercept determines the binding constant.

Thermodynamic parameters: The thermodynamic parameters such as change in free energy $(\Delta G)$, the entropy change $(\Delta S)$ and the enthalpy change $(\Delta \mathrm{H})$ for any particular interaction can be determined by using fluorescence quenching data. By applying equation 5 , it is possible to determine binding constants at various temperatures, with fluorescence quenching measurements.

The different binding constants corresponding to different temperatures can be given by Van't Hoff equation [20-25],

$$
\ln K=-\frac{\Delta H}{R T}+\frac{\Delta S}{R}
$$

Where, $K$ is binding constant, $\mathrm{R}$ is the gas constant, $\mathrm{T}$ is absolute temperature, $\Delta \mathrm{H}$ is the change in enthalpy and $\Delta \mathrm{S}$ is the change in entropy accompanying the molecular interaction.

Subsequently, free energy change at different temperatures can be obtained by Gibb's equation,

$$
\Delta \mathrm{G}=\Delta \mathrm{H}-\mathrm{T} \Delta \mathrm{S}
$$

Thus, a fluorescence study helps to get an idea about the calculations of thermodynamic parameters. The nature of binding forces can be predicted by observing these thermodynamic parameters. The acting forces between the different molecules include hydrogen bond, van der Waals' forces, electrostatic interactions and hydrophobic interaction. Ross summed up the thermodynamic laws [37] for estimating the type of the binding force between organic micromolecule and biological 
Citation: Bhopate DP, Kim K-H, Mahajan PG, Gore AH, Patil SR, et al. (2017) Fluorescent Chemosensor for Quantitation of Multiple Atmospheric Gases. J Nanomed Nanotechnol 8: 436. doi: 10.4172/2157-7439.1000436

Page 8 of 9

macromolecule. If both $\Delta \mathrm{H}$ and $\Delta \mathrm{S}$ are positive, the main force will be a hydrophobic force while if both are negative, then hydrogen bond and van der Waals' forces will be key forces of interaction and reaction mainly enthalpy driven. Also, if $\Delta \mathrm{H} \approx 0$ and $\Delta \mathrm{S}$ have positive value then there will be an electrostatic force between the acting molecules. The negative value of $\Delta \mathrm{G}$ will indicate the spontaneous nature of reaction and vice-versa [21-25].

Applications of the proposed fluorimetric method for the determination of gas (specific) in environmental (atmospheric) samples: The method of sensing of gas by fluorescent sensor based on fluorescence off/on approach was used to develop fluorimetric method of detection of gas in atmospheric sample collected from local region. The atmospheric sample collected with help of air sampler from local or polluted region were spiked with standard gas at two different concentration levels, then diluted to bring gas concentration within the working linear range and analyzed with the method proposed via a standard addition method [22-24]. The accuracy and reliability of the method was further ascertained by recovery studies via a standard addition.

\section{Comparison of Gas Sensing Methods}

In this review, the authors have tried to give information to the researchers engaged in establishing analytical profiles of the all sensors in comparison with fluorimetric method. The fluorimetric method has the advantages of lower detection limit (LOD) and wider linear range of concentration for gases over the existing sensing methods is illustrated in Table 1.

\section{Conclusion}

In this review, a large number of different approaches for the development of new materials for fluorescent gas sensing have been discussed. In this discussion the design/preparation of material especially organic molecules/their nanoparticles by reprecipitation method and their characterization is one part. The studies of photo physical behavior to explore suitability of fluorescent materials for sensing and detection based of analyte gas molecules on fluorescence on-off approach and development of analytical method for real sample analysis (atmospheric gaseous sample) are the other parts of are cover by this review. From the examples published in literature, a simple fluorimetric-assay visualizes the presence of gas and permits quantitation of its amount over the whole concentration range $(0-100 \%)$. It is free of the problems often encountered in the conventional gas-sensing systems. Our review is particularly appealing to the mega sciences with huge societal impacts, such as volcanology and seismology, where field tracking of the gas mixtures with high hazardous gas contents plays a crucial role in disaster prediction and prevention.

The rapid, robust, simple and efficient assay with the long-term goal to reach a new technology for real atmospheric gases quantitation is the outcome of present review.

\section{Acknowledgment}

The authors are thankful to the University Grants Commission (UGC), New Delhi for research funding of the project no. F. No. 41-217/2012 (SR) and a research fellowship with one of the author DPB. We are also thankful to the Department of Science and Technology (DST), New Delhi for providing funds to the Department of Chemistry, Shivaji University, Kolhapur under the FIST-Level-1 program for infrastructure improvement and UGC New Delhi for grants under the program UGC-DRS-I. Also thanks to BK21 plus program and NRF grant funded by the Korea Government (BRL 2015042417).

\section{References}

1. Amao Y, Nakamura N (2005) An optical sensor with the combination of colorimetric change of $\alpha$-naphtholphthalein and internal reference luminescent dye for $\mathrm{CO}_{2}$ in water. Sensors and Actuators B: Chemical 107: 861-865.

2. Amornthammarong N, Zhang, JZ (2008) Hybrid Fluorometric Flow Analyzer for Ammonia. Anal Chem 80: 1019-1026.

3. Basabe-Desmonts L, Reinhoudt DN, Crego-Calama M (2007) Design of fluorescent materials for chemical sensing. Chem Soc Rev 36: 993-1017.

4. Beck K, Kunzelmann T, Schickfus MV, Hunklinger S (1999) Contactless surface acoustic wave gas sensor. Sensors and Actuators B: Chemical 76: 103-106.

5. Bhopate DP, Mahajan PG, Kolekar GB, Garadkar KM, Patil SR (2015) Polyvinyl pyrrolidone capped fluorescent anthracene nanoparticles for sensing fluorescein sodium in aqueous solution and analytical application for ophthalmic samples. Luminescence 30: 1055-1063.

6. Bhopate DP, Mahajan PG, Garadkar KM, Kolekar GB, Patil SR (2014) Pyrene nanoparticles as a novel FRET probe for detection of rhodamine 6G spectroscopic ruler for textile effluent. RSC Adv 4: 63866-63874.

7. Bhopate DP, Mahajan PG, Garadkar KM, Kolekar GB, Patil SR (2015) A highly selective and sensitive single click novel fluorescent off-on sensor for copper and sulfide ions detection directly in aqueous solution using curcumin nanoparticles. New J Chem 39: 7086.

8. Bhopate DP, Mahajan PG, Garadkar KM, Kolekar GB, Patil SR (2013) Cetyltrimethylammonium bromide stabilized perylene nanoparticles for fluorimetric estimation of bicarbonate $\left(\mathrm{HCO}_{3}^{-}\right)$anion: spectroscopic approach Anal. Methods 5: 5324-5330.

9. Bhopate DP, Mahajan PG, Patil AA, Salunkhe RS, Garadkar KM, et al. (2017) A quinazolinone based novel fluorescent nanoprobe for selective detection of Bovine serum albumin: Spectroscopic, photophysical and analytical approach Imperial Journal of Interdisciplinary Research (IJIR) 3: 376-390.

10. Bradley CC (2003) Charge transfer from ammonia physisorbed on nanotubes Phys Rev Lett 91: 218301-218304.

11. Currie JF, Essalik A, Marusic JC (1999) Micromachined thin film solid state electrochemical $\mathrm{CO}_{2}, \mathrm{NO}_{2}$ and $\mathrm{SO}_{2}$ gas sensors. Sensors and Actuators $\mathrm{B}$ : Chemical 59(2-3): 235-241.

12. Crank J (1975) The mathematics of diffusion, by Brunel University, Uxbridge (2ndedn) Clarendon Press, Oxford, ISBN 0198533446

13. Dansby-Sparks RN, Jin J, Mechery SJ, Sampathkumaran U, Owen TW, e al. (2010) Fluorescent-dye-doped sol-gel sensor for highly sensitive carbon dioxide gas detection below atmospheric concentrations. Anal Chem 82 593-600.

14. Ding D, Li K, Liu B, Tang BZ (2013) Bioprobes based on AlE Fluorogens. Acc Chem Res 46: 2441

15. Dorozhkin LM, Rozanov IA (2001) Acoustic Wave Chemical Sensors for Gases. Journal of Analytical Chemistry 56: 399-416.

16. Firth JG, Jones A, Jones $T$ (1973) The principles of the detection of flammable atmospheres by catalytic devices. Combustible Flame 20: 303-311.

17. Guo ZQ, Song NR, Moon JH, Kim M, Jun EJ, et al. (2012) Benzobisimidazoliumbased fluorescent and colorimetric chemosensor for $\mathrm{CO}_{2}$. J Am Chem Soc 134 17846.

18. Gillanders RN, Morawska PO, Nguyen H, Chen F, Campbell IA, et al. (2015) Organic Semiconductor Centre, SUPA, School of Physics \& Astronomy University of St Andrews, St Andrews, Fife KY16 9SS, Scotland.

19. Hong YN, Lam YW, Tang BZ (2011) Aggregation-induced emission. Chem Soc Rev 40: 5361

20. Hu RR, Leung NLC, Tang BZ (2014) AIE macromolecules: syntheses, structures and functionalities. Chem Soc Rev 43: 4494

21. Hubert T, Boon-Brett L, Black G, Banach U (2011) Hydrogen sensors - A review. Sensors and Actuators B: Chemical 157: 329-352.

22. Huijuan $Y$, Haibing $L$ (2010) Urea type of fluorescent organic nanoparticles with high specificity for $\mathrm{HCO}_{3}{ }^{-}$anions. Sens. Actuators B 148: 81-86.

23. Ishida M, Kim P, Choi J, Yoon J, Kim D, Sessler JL (2013) Benzimidazoleembedded $\mathrm{N}$-fused aza-indacenes: synthesis and deprotonation-assisted optical detection of carbon dioxide. Chem Commun 49: 6950. 
Citation: Bhopate DP, Kim K-H, Mahajan PG, Gore AH, Patil SR, et al. (2017) Fluorescent Chemosensor for Quantitation of Multiple Atmospheric Gases. J Nanomed Nanotechnol 8: 436. doi: 10.4172/2157-7439.1000436

24. Jones E (1987) Solid State Gas Sensored Moseley P T and Tofield B C (Bristol: Adam-Hilger) 2: 17-31

25. Korotcenkov G (2007) Practical aspects in design of one-electrode semiconductor gas sensors: Status report. Sensors and Actuators B: Chemical 121: 664-678.

26. Lin J (2000) Recent development and applications of optical and fiber-optic pH sensors. Trac-Trends Anal Chem 19: 541.

27. Liu Y, Tang Y, Barashkov NN, Irgibaeva IS, Lam J, et al. (2010) Fluorescent chemosensor for detection and quantitation of carbon dioxide gas. J Am Chem Soc 132: 13951-13953.

28. Lakowicz JR (2006). Principles of fluorescence spectroscopy. (3rdedn), Plenum Press, New York, USA.

29. Ma Y, Xu H, Zeng Y, Ho CL, Chui $\mathrm{CH}$, et al. (2015) A charged iridophosphor for time-resolved luminescent $\mathrm{CO}_{2}$ gas identification. J Mater Chem C 3: 66.

30. Ma Y, Xu H, Zeng Y, Ho CLC, Chui $\mathrm{CH}$, et al. (2015) A water-soluble tetraphenylethene based probe for luminescent carbon dioxide detection and its biological application. J Mater Chem C 3: 11850.

31. Mills A, Hodgen S (2005) Advanced Concepts in Fluorescence SpectroscopyPart A Small Molecule Sensing, Topics in Fluorescence Spectroscopy. Springer, Berlin 9: 119.

32. Modi A (2003) Miniaturized gas ionization sensors using carbon nanotubes. Nature 424: 171-174.

33. Neethirajan S, Jayas DS, Sadistap S (2009) Carbon Dioxide $\left(\mathrm{CO}_{2}\right)$ Sensors for the Agri-food Industry-A Review. Food Bioprocess Technol 2: 115

34. An BK, Kwon, SK, Jung SD, Park SY (2002) Enhanced Emission and Its Switching in Fluorescent Organic Nanoparticles. J Am Chem Soc 124: 14410 14415.

35. Peng Q, Yi Y, Shuai Z, Shao J (2007) Toward quantitative prediction of molecular fluorescence quantum efficiency: Role of Duschinsky rotation. J Am Chem Soc 129: 9333.

36. Postek MT, Vladar AE (2001) Critical-Dimension Metrology and the SEM Handbook of Silicon Semiconductor Metrologyed. (New York: CRC Press) 295-334

37. Ross PD, Subramanian S (1981) Thermodynamics of protein association reactions: forces contributing to stability. Biochem 20: 3096-102.

38. Shayu L, Liming H, Fei X, Yi L, Guoqiang Y (2004) Enhanced fluorescent emission of organic nanoparticles of an intramolecular proton transfer compound and spontaneousformation of one-dimensional nanostructures. J Phys Chem B 108: 10887-10892.
39. Sun X (2015) Design, synthesis and evaluation of fluorescent sensors for the detection of saccharide and reactive oxygen species: A thesis submitted for the degree of Doctor of Philosophy, University of Bath, Department of Chemistry.

40. Tardy P, Coulon JR, Lucat C, Menil F (2004) Dynamic thermal conductivity sensor for gas detection. Sensors and Actuators B: Chemical 98: 63-68.

41. Velasco-Garcia N, Valencia-Gonzalez MJ, Diaz-Garcia ME (1997) Fluorescent organofilms for oxygen sensing in organic solvents using a fiber optic system. Analyst 122: 1405-1409.

42. Wang J, Karpus J, Zhao BS, Luo Z, Chen PR, et al. (2012) A selective fluorescent probe for carbon monoxide imaging in Living cells. Angew Chem Int Ed 51: 9652-9656.

43. Wang LY, Dong L, Wang L (2004) Application of organic nanoparticles as fluorescence probe in the determination of nucleic acids. Anal Lett 37: 1811.

44. Willimams RT, Birdges JW (1964) Fluorescence of solutions: A review. J Clin Path 17: 371.

45. Wolfbeis OS, Weis LJ, Leiner MJP, Ziegler WE (1988) Fiber-optic fluorosensor for oxygen and carbon dioxide. Anal Chem 60: 2028.

46. Xu QL, Lee SY, Kim MH, Bouffard J, Yoon J (2013) Polydiacetylene-Based Colorimetric and Fluorescent Chemosensor for the Detection of Carbon Dioxide. J Am Chem Soc 135: 17751.

47. Xu W, McDonough RC, Langsdorf B, Demas JN, DeGraff BA (1994) Oxygen Sensors Based on Luminescence Quenching: Interactions of Metal Complexes with the Polymer Supports. Anal Chem 66: 4133-4141.

48. Yanai N, Kitayama K, Hijikata Y, Sato H, Matsuda R, et al. (2011) Gas detection by structural variations of fluorescent guest molecules in a flexible porous coordination polymer. Nature Materials 10: 787-793.

49. Yunusa Z, Hamidon MN, Kaiser A, Awang Z (2014) Gas sensors: A review Sensors and Transducers 168: 61-75.

50. Shen G, Wang W, Yang Y Ding, Russell AG (2011) Emissions of PAHs from indoor crop residue burning in a typical rural stove: emission factors, size distributions, and gas particle partitioning. 45: 1206-1212.

51. Zhan XQ, Li DH, Zheng H, Xu JG, Zhou YQ (2002) Fluorimetric determination of nitrogen oxides in the air by a novel red-region fluorescent reagent. Talanta 58: $855-860$.

52. Zhao Z, Lu P, Lam JWY, Wang Z, Chan CYK, et al. (2011) Molecular anchors in the solid state: Restriction of intramolecular rotation boosts emission efficiency of luminogen aggregates to unity. Chem Sci 2: 672. 